\title{
RUNOUT ANALYSIS IN FATIGUE INVESTIGATION
}

\author{
S. Sarkani ${ }^{1}$, T. A. Mazzuchi ${ }^{2}$, D. Lewandowski ${ }^{3}$, and D. P. Kihl ${ }^{4}$ \\ ${ }^{1}$ Department of Engineering Management \& Systems Engineering, The George Washington University, U.S.A. \\ ${ }^{2}$ Department of Engineering Management \& Systems Engineering, George Washington University, Washington, DC 20052 \\ ${ }^{3}$ University of Technology, Delft, The Netherlands \\ ${ }^{4}$ Structures \& Composites Division, Naval Surface Warfare Center, West Bethesda, MD 20817-1956
}

\section{ABSTRACT}

Experiments to evaluate and validate processes of engineering and science are particularly important when the phenomena under investigation may be studied empirically, as can metal fatigue or medical injection dosages. Sometimes during an experiment, individual tests in a series are not completed, or must be suspended or interrupted, or are somehow damaged. Such test points are referred to as "suspended," or "censored points," or in the case of metal fatigue are simply called runouts. All these names refer to test specimens that do not fail under the same conditions as do other specimens or data points. In reliability and biometry, runout data are termed Type I censored observations. Inclusion of runouts in data analysis and interpretation can be problematic, and such ad hoc approaches as ignoring the runout observation or treating it as a failure can significantly affect estimation. Even when data are plotted and represented by a mathematical function utilizing least squares analysis to minimize data scatter about the "best fit" function, runouts and censored observations cause estimation problems because they cannot be incorporated directly into a leastsquares analysis procedure. This paper offers a methodology to help alleviate the problems presented by runouts and censored data. The methodology handles runouts and censored data by employing the maximum likelihood estimation (MLE) method to incorporate the censored data properly. The methodology is illustrated with an example problem.

\section{INTRODUCTION}

We consider a set of data from fatigue tests such as are typically conducted on a batch of identical specimens, each cycled under constant amplitude stress, $S$, until the specimen fails. At failure, the number of cycles applied, $N$, is recorded. At a given constant amplitude stress level, we assume that $N$ is given by the Weibull distribution $N \sim W e i(\alpha, \beta)$. Then $X=\ln (N)$ and thus $N=e^{X}, d N=e^{X} d X$. The extreme value distribution is denoted $X \sim E V(\mu, \sigma)$. To calculate the mean and variance of $X \sim E V(\mu, \sigma)$, we calculate the moment generating function

$$
\mathrm{E}\left[e^{t X}\right]=\int_{-\infty}^{+\infty} e^{t x} \frac{1}{\sigma} e^{\left(\frac{x-\mu}{\sigma}\right)-e^{\left(\frac{x-\mu}{\sigma}\right)}} d x
$$

Using eqn (1), we derive

$$
\begin{aligned}
\mathrm{E}[X] & =\left.\left\{\mu e^{t \mu} \Gamma(t \sigma+1)+\sigma e^{t \mu} \Gamma^{\prime}(t \sigma+1)\right\}\right|_{t=0} \\
& =\mu \Gamma(1)+\sigma \Gamma^{\prime}(1)=\mu+\sigma \Gamma^{\prime}(1)
\end{aligned}
$$

where $\Gamma^{\prime}(1)=-\gamma$ and $\gamma$ is Euler's constant $\gamma \approx 0.5772$. Then,

$$
\left.\mathrm{E}\left[X^{2}\right]=\operatorname{Var}(X)=\sigma^{2} \mid \Gamma^{\prime \prime}(1)-\left(\Gamma^{\prime}(1)\right)^{2}\right]
$$

and $\Gamma^{\prime \prime}(1)-\left(\Gamma^{\prime}(1)\right)^{2}=\pi^{2} / 6$, and $\Gamma^{\prime}(\alpha)$ and $\Gamma^{\prime \prime}(\alpha)$ are the first and second deviations of the Gamma Function.

\subsection{Regression model}

The regression $\quad$ model $X \equiv \ln (N)=\ln (k)-m \ln (S)+\sigma \varepsilon, \quad$ where $\quad \varepsilon \sim E V(0,1), \quad$ (thus 
$X \mid S \sim E V(\ln (k)+m \ln (S), \sigma))$, is widely used in reliability and biometry (Lawless [1], Hosmer [2]). We write the density of $X|S=\ln (N)| S$ and the corresponding reliability function

$$
\begin{aligned}
& f(x \mid S, k, m, \sigma)=\frac{1}{\sigma} e^{\left(\frac{x-\ln (k)+m \ln (s)}{\sigma}\right)-e^{\left(\frac{x-\ln (k)+m \ln (s)}{\sigma}\right)}} \\
& R(x \mid S, k, m, \sigma)=e^{-e^{\left(\frac{x-\ln (k)+m \ln (S)}{\sigma}\right)}}
\end{aligned}
$$

These can be used to construct the likelihood function for the MLE.

\subsection{The likelihood function}

In such introductory texts on statistics as Bedford [3], the likelihood that a set of parameters $\theta$ will be found is defined for a series $x_{1}, x_{2}, \ldots, x_{n}$ of independent observations as

$$
L\left(\theta ; x_{1}, x_{2}, \ldots, x_{n}\right)=\prod_{i=1}^{n} f\left(x_{i} \mid \theta\right)
$$

where $f$ is the density function of $X$; however, this assumes that complete observations, e.g., all failure times, can be made. When Type I censoring occurs at a time $x^{*}$, and $r$ items have failed and $n-r$ are removed from the test without failure, the likelihood is redefined and can be shown to be equal to

$$
\begin{aligned}
& L(k, m, \sigma ; D)= \\
& =\left(\frac{1}{\sigma}\right)^{\sum_{j=1}^{h} r_{j}} \exp \left\{\sum_{j=1}^{h} \sum_{i=1}^{r_{j}}\left(\frac{x_{i j}-\ln (k)+m \ln \left(S_{j}\right)}{\sigma}\right)-\sum_{j=1}^{h} \sum_{i=1}^{r_{j}} e^{\left(\frac{x_{i j}-\ln (k)+m \ln \left(S_{j}\right)}{\sigma}\right)}-\sum_{j=1}^{h}\left(l_{j}-r_{j}\right) e^{\left(\frac{x_{j}^{*}-\ln (k)+m \ln \left(S_{j}\right)}{\sigma}\right)}\right\}
\end{aligned}
$$

Optimizing $L$ is equivalent to optimizing $\mathcal{L}=\ln (L)$, which is given by eqn (3), and can be maximized using a Newton-Raphson technique:

$$
\begin{aligned}
\ln (L(k, m, \sigma ; D)) & =-\sum_{j=1}^{h} r_{j} \ln (\sigma)+\sum_{j=1}^{h} \sum_{i=1}^{r_{j}}\left(\frac{x_{i j}-\ln (k)+m \ln \left(S_{j}\right)}{\sigma}\right) \\
& -\sum_{j=1}^{h} \sum_{i=1}^{r_{j}} e^{\left(\frac{x_{i j}-\ln (k)+m \ln \left(S_{j}\right)}{\sigma}\right)}-\sum_{j=1}^{h}\left(l_{j}-r_{j}\right) e^{\left(\frac{x_{j}^{*}-\ln (k)+m \ln \left(S_{j}\right)}{\sigma}\right)}
\end{aligned}
$$

\section{APPLICATION OF THE MODEL AND INITIAL RESULTS}

We demonstrate the usefulness of the Weibull regression model developed here using a hypothetical data set. Nine specimens are tested at 3 different stress levels. One specimen does not fail when reaching the age of 100,000 time units, and is thus classified as a runout. The likelihood function, eqn (2), is unimodal (see Figure 1).

To find the MLEs of $\theta=\{\ln (k), m, \sigma\}$ by applying the Newton-Raphson method, we need three equations, which would be functions of this vector of variables. Unfortunately, the NewtonRaphson algorithm does not converge for this data set. Therefore, a different approach is applied. We fix $\sigma$, and then use the Newton-Raphson method to calculate the MLEs of $\ln (k)$ and $m$ such that the maximum of the likelihood function is a one-dimensional function of $\sigma$, which many available algorithms can maximize. Hence we reduce the problem from three dimensions to one. This approach works well and gives accurate results that are exactly the same as those returned by 

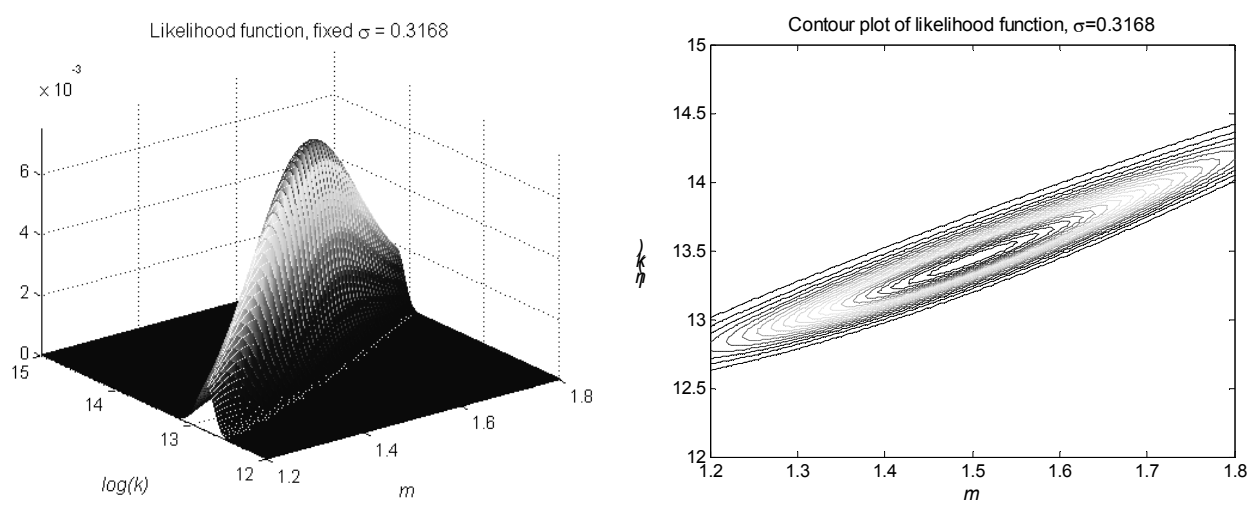

Figure 1: Plot of the likelihood function $L$.

the Nelder-Mead simplex search method, even though the starting points were fairly far from the optimal solution. The initial guesses for $\ln (k)$ and $m$ are determined by considering the runout as a failure and then applying the standard least-squares error method, but there is no need for a very accurate approximation. For the parameter $\sigma$, the unbiased estimate of the standard deviation of error term $\sigma_{0}$ is taken as the initial guess,

$$
\sigma_{0}=\sqrt{\frac{1}{l-1} \sum_{j=1}^{h} \sum_{i=1}^{l_{j}}\left(x_{i j}-\left(\ln \left(k_{s}\right)-m_{s} \ln \left(S_{j}\right)\right)\right)^{2}}
$$

where $\ln \left(k_{s}\right)$ and $m_{s}$ are the least-squares error estimators. The Newton-Raphson method allows the following MLEs for the model's input parameters to be determined: $\ln \left(k_{m}\right)=13.438, m_{m}=$ $1.499, \sigma_{m}=0.3168$. Given these values, we plot the estimated $\mathrm{S} / \mathrm{N}$ curve obtained using both the least-squares error and the MLE methods.

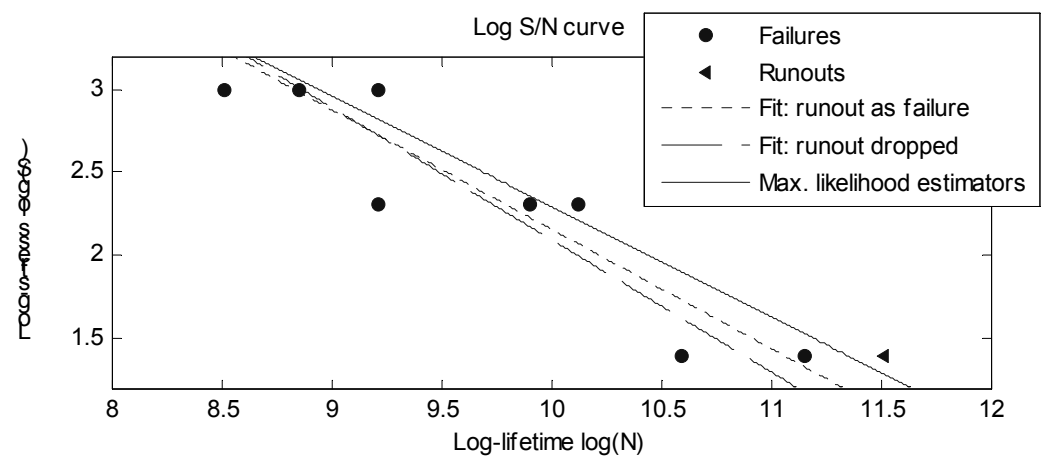

Figure 2: Estimations of the $\mathrm{S} / \mathrm{N}$ curve.

Clearly, the S/N curves corresponding to the MLEs take higher values than the curves obtained using the least-squares error technique. This result is expected, because we have now taken into account, that one of the specimens did not fail during the test or during its residual life, and that the mean lifetime is longer than the estimation obtained by considering the runout as a 
a) Runout as failure

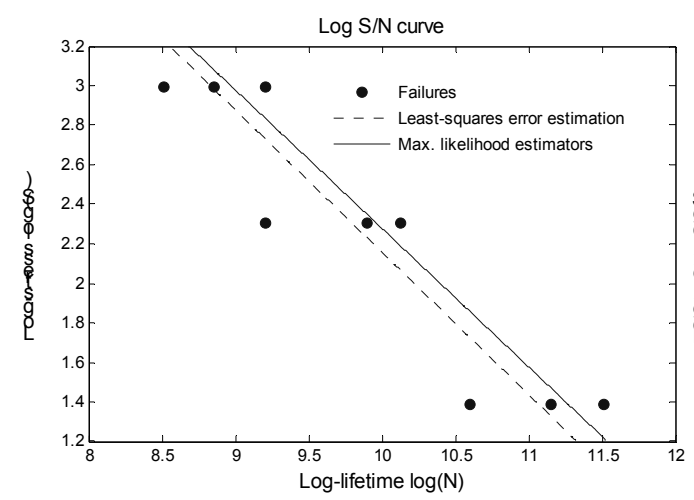

b) Runout dropped

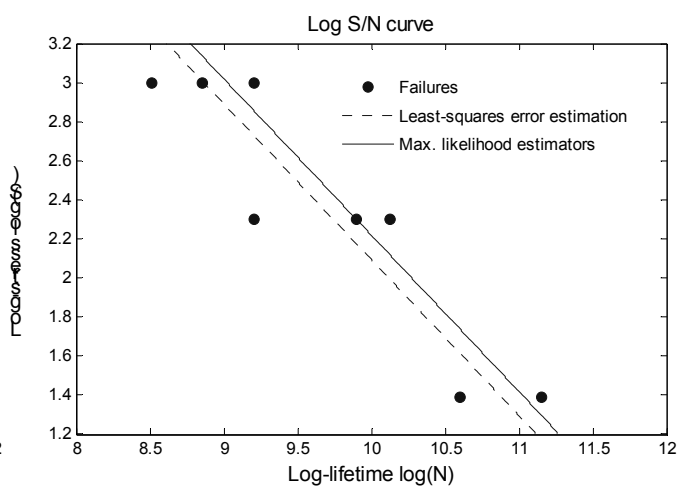

Figure 3: Comparison of S/N Curves obtained by two different techniques.

failure or by not taking this specimen into consideration at all. The difference between the estimated $\mathrm{S} / \mathrm{N}$ curves is proportional to the probability that the specimen whose observation was interrupted survives longer than the termination time of the test. We investigate two cases: (1) Runout as failure and (2) Runout dropped, and show (3) Bounds on the prediction.

\subsection{Runout as failure}

We select this case to allow direct comparison of the least-squares error and the MLE methods by considering the data set as complete observations. We alter the data set such that the termination time is regarded as the lifetime of the component whose test is terminated before the actual failure. We apply both the Newton-Raphson and the Nelder-Mead simplex methods to confirm the results. The MLEs here are $\ln (k)=13.247, m=1.427$ and $\sigma=0.2825$. Figure 3a shows a rather big difference between the $\mathrm{S} / \mathrm{N}$ curves obtained by applying the two methods of estimation presented above (ignoring the runout and treating it as a failure). The MLE results tend to imply that the tested specimens have higher resistance to stress than do the runout specimens. The difference is caused by the underlying assumptions that we implicitly or explicitly introduced into the analysis by choosing a specific method of parameter estimation. The least-squares error method is fully nonparametric, whereas the MLE approach requires that an underlying probability distribution be specified for the specimen's lifetime. The latter method can lead to incorrect results if the true underlying probability distribution is different from the assumed one. The Weibull distribution is one of those most widely used in reliability, and an enormous number of applications have proven its usefulness. Moreover, a parametric model can handle outliers much better than a nonparametric model can, because it assigns a smaller probability of occurrence to such observations. The least-squares method treats all observations equally; hence outliers introduce large variances into the estimation.

\subsection{Runout dropped}

Consider the situation when the runout is completely removed from the data set and compare the least-squares error and the MLE methods. The MLEs in this case are $\ln (k)=12.77, m=1.249$, and $\sigma=0.2627$. Figure 3 a shows both estimations of the $\mathrm{S} / \mathrm{N}$ curve. Again, the MLE indicates that the expected life of the specimen in terms of stress is longer than the least-squares error estimate. In 
general, the observations lie below the S/N curve given by the MLE. This might be because the observations with longer lifetimes contribute more to the overall likelihood function.

\subsection{Bounds on the prediction}

Under the assumption that the logarithms of the observation times are outcomes of the extreme value distribution, we can determine a $90 \%$ prediction interval for the life, as well as the conditional probability density functions. Both features are added to the plot in Fig. 3a. Moreover, because the errors in the least-squares fitting method are normally distributed, we can plot the $95 \%$ confidence interval for the estimated life. Fig. $3 \mathrm{~b}$ shows the least-squares error estimate of the $\mathrm{S} / \mathrm{N}$ curve with the confidence bounds when the runout is treated as a failure.

a)

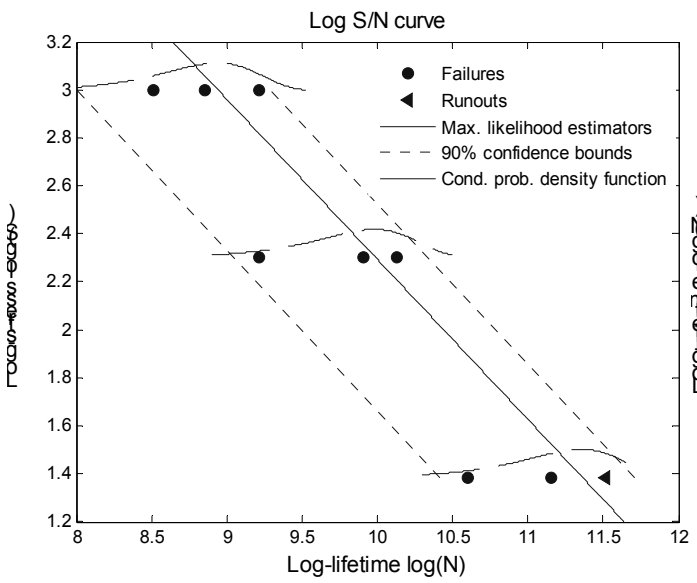

b)

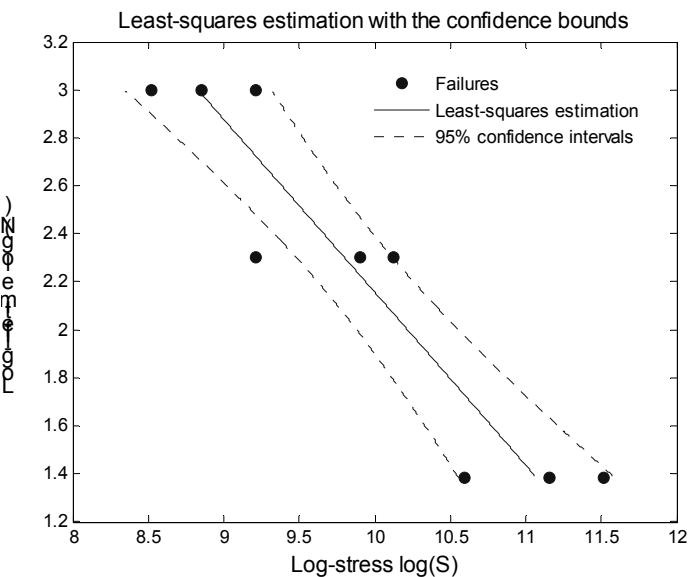

Figure 4: More featured plots of the estimation of the $\mathrm{S} / \mathrm{N}$ curve.

We derive confidence bounds on the parameters $\theta_{m}=\left\{\ln \left(k_{m}\right), m_{m}, \sigma_{m}\right\}$ by applying the likelihood ratio test

$$
\begin{gathered}
-2 \ln \left(\frac{L(k, m, \sigma ; D)}{L\left(k_{m}, m_{m}, \sigma_{m} ; D\right)}\right)=\chi_{\alpha, 1}^{2} \\
\Downarrow \\
L(k, m, \sigma ; D)-L\left(k_{m}, m_{m}, \sigma_{m} ; D\right) \cdot e^{-\frac{\chi_{\alpha, 1}^{2}}{2}}=0
\end{gathered}
$$

where $L(k, m, \sigma ; \mathcal{D})$ is the maximum of the likelihood function given in eqn (2). We need to find the isosurface described by eqn (4). We take 1 (one) as the degree of freedom of the chi-square distribution in this equation so that we change only one parameter at a time, keeping the other two constant. This isosurface, along with its projections on planar planes, is depicted in Figure 5. 


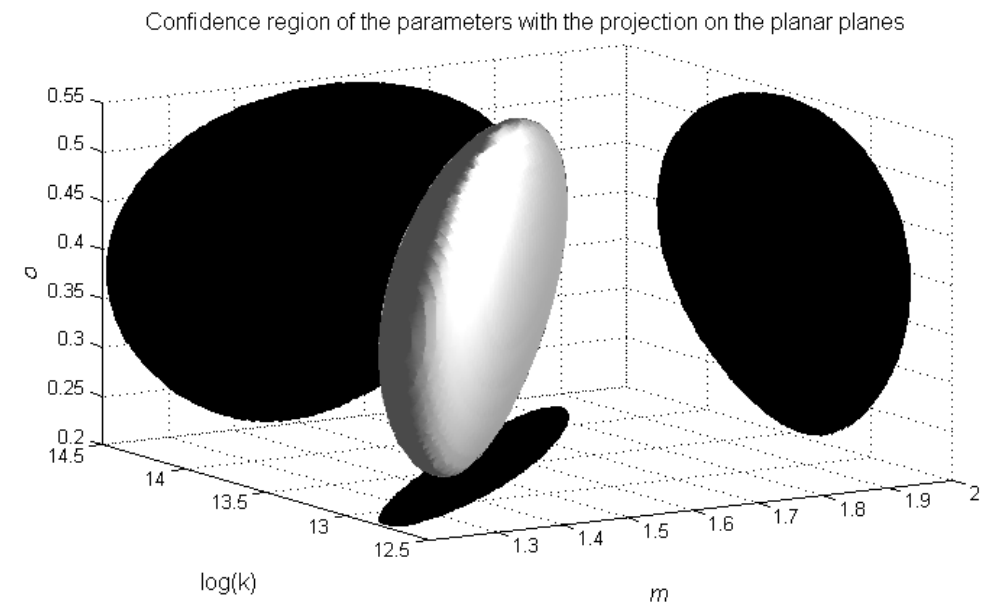

Figure 5: The 95\% confidence region of the parameters.

\section{CONCLUSIONS}

When analysts face the problem of dealing with censored data, treating those observations as failures is inappropriate, because such interference adds information into the data set that was not revealed by the specimens under the test itself. On the other hand, excluding censored observations from the analysis is clearly a waste of time and money spent on testing a given specimen. A natural way to deal with such a data set is to use the MLE method. The only drawback to using MLE is that the analyst must assume an underlying probability distribution function for the lifetime of the tested specimens, which in general would depend on the nature of the items being tested. Many applications would justify use of the Weibull distribution. Using a hypothetical data set, we show that the MLE approach can be applied successfully. Supported by the NewtonRaphson method, our method is an attractive way to derive the $\mathrm{S} / \mathrm{N}$ curve because it depends only on the observed data and on the underlying probability distribution, and because it makes use of all the information included in the data.

\section{REFERENCES}

[1] Lawless, J. F., Statistical Models and Methods for Lifetime Data, Wiley, New York, 1982.

[2] Hosmer, D. W. and Lemeshow, S., Applied Survival Analysis-Regression Modeling of Time to Event Data, Wiley, New York, 1999.

[3] Bedford, T. and Cooke, R. M., Probabilistic Risk Analysis-Foundations and Methods, Cambridge University Press, UK, 2001. 